\title{
The Pro-Inflammatory Effect of Staphylokinase Contributes to Community-Associated Staphylococcus Aureus Pneumonia
}

\section{Yanan Wang}

Department of Laboratory Medicine, Ren Ji Hospital, School of Medicine, Shanghai Jiao Tong University, Shanghai 200127, China

\section{$\mathrm{Na}$ Zhao}

Shanghai Jiao Tong University

\section{Ying Jian}

Shanghai Jiao Tong University

\section{Yao Liu}

Shanghai Jiao Tong University

\section{Lin Zhao}

Shanghai Jiao Tong University

\section{Lei He}

Shanghai Jiao Tong University https://orcid.org/0000-0003-4679-5462

\section{Qian Liu}

Shanghai Jiao Tong University

Min Li (D ruth_limin@126.com )

Shanghai Jiao Tong University https://orcid.org/0000-0002-8571-2909

\section{Article}

Keywords: Community-associated Staphylococcus aureus, Staphylokinase, Pneumonia, Excessive inflammation, NLRP3 inflammasome

Posted Date: November 22nd, 2021

DOI: https://doi.org/10.21203/rs.3.rs-1084745/v1

License: (c) (1) This work is licensed under a Creative Commons Attribution 4.0 International License. Read Full License

Version of Record: A version of this preprint was published at Communications Biology on June 23rd, 2022. See the published version at https://doi.org/10.1038/s42003-022-03571-x. 

1 The pro-inflammatory effect of Staphylokinase contributes to

2 community-associated Staphylococcus aureus pneumonia

3 Yanan Wang ${ }^{1 \#}, \mathrm{Na}_{\text {Zhao }}{ }^{1 \#}$, Ying Jian ${ }^{1}$, Yao Liu ${ }^{1}$, Lin Zhao ${ }^{1}$, Lei He ${ }^{1}$, Qian

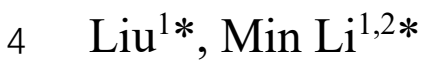

5

6 1. Department of Laboratory Medicine, Ren Ji Hospital, School of Medicine, Shanghai

7 Jiao Tong University, Shanghai 200127, China.

8 2. Faculty of medical laboratory science, Shanghai Jiaotong University School of

9 Medicine, Shanghai 200127, China.

10

11 \# These authors contributed equally to this work.

* Corresponding author:

Min Li. Email: ruth_limin@126.com, 86-21-68383297; Medicine, No. 1630 East Road, Pudong New Area, Shanghai

Qian Liu. Email: qq2005011@,163.com,86-21-68383614; Medicine, No. 1630 East Road, Pudong New Area, Shanghai

Postal address: Renji Hospital Affiliated to Shanghai Jiaotong University School of

Postal address: Renji Hospital Affiliated to Shanghai Jiaotong University School of 


\section{The pro-inflammatory effect of Staphylokinase contributes to} community-associated Staphylococcus aureus pneumonia

\section{Abstract}

Pneumonia caused by community-associated Staphylococcus aureus (CA-SA) has high morbidity and mortality. Pathogens can express a variety of virulence factors to promote infection. The game between the host immune system and pathogens determines the degree of infection and tissue damage, but its mechanism has not been well explored. In this study, we found that staphylokinase (SAK) is highly expressed in CA-SA lineage ST398 and promotes lung infection in both wild-type and cathelicidins-related antimicrobial peptide CRAMP knockout $\left(C R A M P^{-/-}\right)$mice. Furthermore, our results showed that SAK can activate the host's innate immune inflammatory response. Importantly, SAK can promote the activation of NLRP3 inflammasome by increasing the production of ROS. This may be one of the important mechanisms of SAK aggravating CA-SA pneumonia after excluding its function of promoting fibrinolysis and neutralizing CRAMP. Our results emphasize the importance of controlling inflammation in acute lung infections, and also provides new insights into the pathogenesis of highly virulent CA-SA.

\section{Keywords}

Community-associated Staphylococcus aureus, Staphylokinase, Pneumonia, Excessive inflammation, NLRP3 inflammasome 


\section{Introduction}

Staphylococcus aureus (S. aureus) is one of the most important pathogens and can cause a wide variety of infections ${ }^{1,2}$. During the past decade, $S$. aureus has become an important cause of community acquired pneumonia ${ }^{3-5}$. In contrast with hospitalassociated S. aureus (HA-SA), which usually infects patients with impaired immunity, community-associated S. aureus (CA-SA) infections can occur in other healthy individuals, suggesting that these bacterial strains have greater virulence ${ }^{6}$.

High expression of virulence genes that promote invasive infections plays an important role in the pulmonary pathology associated with CA-SA related pneumonia ${ }^{5,7,8}$. However, excessive local inflammation and tissue damage can also impede bacterial clearance ${ }^{9}$. Mouse pneumonia models lacking innate immune signal components showed significantly improved outcomes when infected by $S$. aureus ${ }^{10,11}$. The NLRP3 inflammasome is essential for the host's immune defense against bacterial, fungal and viral infections ${ }^{12}$. But, when NLRP3 is dysregulated or overactivated, the activated caspase-1 promotes the maturation of IL-1 $\beta$ and IL-18, facilitates the recruitment of neutrophils and participates in the inflammatory process of acute lung injury damage ${ }^{9,13-15}$.

The ST398 clone was previously considered to be livestock-associated, but more and more epidemiological studies have shown that ST398 isolates spread in community populations are usually not related to animal infections ${ }^{16}$. The isolation rate of ST398 continues to increase, and it has become one of the most prevalent CA-SA clones in Shanghai, China ${ }^{16-18}$. We have reported the effects of high expression levels of 
virulence genes such as the ESAT-6 secretion system on the pathogenicity of ST398 ${ }^{19,20}$. In addition, prophage also plays an important role in bacterial pathogenicity and evolution $^{21,22}$. The gene encoding staphylokinase (SAK) is located on Prophage $3^{23}$ and it is conserved in human adapted S. aureus $(\mathrm{HO}-\mathrm{SA})^{16}$. SAK was originally found to promote the activation of plasminogen and exert its fibrinolytic function in specific hosts $^{24,25}$. Moreover, studies have shown that SAK can bind to $\alpha$-defensins ${ }^{26}$, human cathelicidin LL-37 and mouse cathelicidins-related antimicrobial peptide (CRAMP) ${ }^{27}$ to regulate fibrinolysis and evade innate immunity defenses. While the research on SAK has often focused on its fibrinolytic function, the role of SAK in the pathogenesis of $S$. aureus remains insufficient and controversial.

In the present study, our results showed increased expression of SAK in ST398 and ST59 isolates, pointing to a potential role of SAK in the virulence of CA-SA. Moreover, we demonstrated a significant role of SAK in the acute ST398 infectious pneumonia model, which is mainly achieved by activating innate immune signals, especially NLRP3 inflammasome-related pathways.

\section{Results}

sak is conserved in HO-SA and is highly expressed in CA-SA

Prophage 3 is considered as a potential molecular marker to distinguish livestockadapted $S$. aureus (LA-SA) from HO-SA ${ }^{28}$. In the present study, we detected the carrying rate of sak in the predominant HA-SA (ST5, ST239), CA-SA (ST59, ST188, ST398) and LA-SA (ST188, ST398, ST97, ST520) isolates of China. The results showed that $96-100 \%$ of HO-SA carried the sak gene, and there was no significant 
difference between HA-SA and CA-SA, while the detection rate of sak in LA-SA was only $0-28 \%$ (Figure 1A). Among these LA-SA, ST398 and ST188, which carry a higher proportion of sak than ST97 and ST520, can be isolated from both humans and animals. These results further confirmed that the carrier rate of sak is related to human infection. Although the sak gene is conserved in HO-SA, we found that the expression level of sak in CA-SA was significantly higher than that in HA-SA (Figure 1B). It has been shown that the increased expression of pathogenicity-related genes is responsible for the high virulence of CA-SA ${ }^{19,20}$. We speculate that the high expression of SAK may also play an important role in promoting the pathogenesis of CA-SA. In addition, we used a chromogenic assay to measure the level of SAK secreted by bacteria in cultures with different incubation times. The results showed that the high SAK secretion level of CA-SA reached its peak in the logarithmic growth phase and remained stable (Figure $1 C)$.

\section{SAK's effect on biofilm formation is plasminogen-dependent}

SAK can promote the activation of human plasminogen and exert its fibrinolytic function. However, SAK can only activate plasminogen in some specific hosts, including humans, rabbits, and sheep, but not mice ${ }^{24}$. Studies in mice expressing human plasminogen showed that SAK can inhibit the formation of biofilms ${ }^{24}$. Biofilms are involved in antibiotic resistance of bacteria and host immune defenses ${ }^{29}$. But the highly virulent $S$. aureus often causes invasive infections ${ }^{19}$ through high expression of virulence-related genes, and their biofilm formation ability is usually weaker than HASA (Figure 1D). 
By detecting the activation effect of SAK on plasminogen, we confirmed the successful construction of sak gene knockout strain and the sak complementary strain (Supplementary Figure 1A). We found that sak deletion did not affect the growth of ST398 and the formation of biofilm in TSB medium (Figure 1E, Supplementary Figure 1B). Furthermore, the addition of SAK protein did not inhibit the formation of biofilms, but after adding human plasma, significant differences were observed between the wildtype and the sak mutant isolates (Figure 1E). This indicates that the ability of SAK to inhibit biofilm formation is achieved primarily by promoting fibrinolysis in specific hosts, and these results are consistent with the research of Kwiecinski et al ${ }^{24}$. Although CA-SA with high SAK expression showed weak biofilm formation ability (Figure 1BD), we speculated that this is more likely due to the high expression level of the important virulence regulator system, such as accessory gene regulator (Agr) system (Supplementary Figure 2A). High expression of the agr locus can inhibit the formation of biofilms ${ }^{30}$, which is consistent with our results (Supplementary Figure 2B). The Agr system positively regulates a variety of virulence-related genes, and the expression of $s a k$ is also regulated by it, but the knockout of sak gene does not affect the expression of Agr system. (Supplementary Figure 2C-D). Therefore, we speculated that the inhibitory effect of SAK on biofilm formation depends on fibrinolysis.

\section{SAK of ST398 can increase the severity of acute lung infection in mice}

It is reported that SAK can directly bind to mouse CRAMP, helping to promote fibrinolysis and evasion of the host's innate immunity ${ }^{27}$, so we used both wild-type and $C R A M P^{-/-}$mice for study. The lung tissues of ST398-infected wild-type mice showed 
obvious congestion and edema (Figure 2A). HE staining of the lung tissues showed that

134 the alveolar structure of mice was destroyed after infection with the ST398 strain, with

135 infiltration of a large number of inflammatory cells (Figure 2A).

136 Immunohistochemistry analysis of lung tissues also proved that ST398-infected mice

137 had more macrophage infiltration (Figure 2B). Furthermore, the wet weight of the lungs

138 and the colony-forming unit (CFU) counts in lung tissues of ST398-infected mice

139 increased significantly (Figure 2C, D). Interestingly, similar results can be seen in

$140 C R A M P^{-/-}$mice (Figure 2E-G). After infection with the sak mutant strain, both wild-

141 type mice and $C R A M P^{-/-}$mice had milder lung pathological changes. Therefore, we

142 speculated that SAK can increase the severity of acute lung infection in mice, and the

143 effect of combining with CRAMP is not the main mechanism of SAK's pathogenicity.

144 The pro-inflammatory effect of SAK in CA-SA pneumonia

145 In order to explore the mechanism of SAK in the process of $S$. aureus infection,

146 we analyzed the RNA sequencing data of lung tissues from wild type and sak gene

147 mutant ST398 infected mice (C57BL/6 wild-type). A total of 580 genes were

148 differentially expressed between the two groups. In addition, the results of GSEA

149 analysis identified that genes related to cytokine response, such as interferon- $\gamma($ IFN- $\gamma$ ),

150 interferon- $\alpha$ (IFN- $\alpha$ ), interleukin-6 (IL-6), interleukin-1 (IL-1), and complement-

151 related genes, and NLRP3 inflammasome-related genes were enriched in the lung

152 tissues of wild-type infected mice (Figure 3A). Moreover, we detected 23 cytokines in

153 the serum of mice, and found that mice infected with wild-type ST398 had higher levels

154 of serum cytokines (Figure 3B). 
In the host's confrontation with pathogens, excessive inflammation can cause tissue damage and poor prognosis. The activation of NLRP3 has been shown to promote cytokine production and cell pyrolysis. We further compared the expression of toll-like receptors, NF- $\mathrm{kB}$ and other NLRP3 inflammasome activation-related genes in the two groups. These molecules are almost all highly expressed in the lung tissues of mice infected with wild-type ST398 (Figure 3C). In addition, we detected the expression of IL-1 $\beta$, IFN- $\alpha$, nlrp3 and caspase- 1 in lung tissues by qRT-PCR, and the results were consistent with RNA sequencing (Figure 3D-G). Combined with the pathological changes in mouse lung tissue, we speculate that the lung tissue of mice infected with wild-type ST398 is in a more active state of inflammatory response than that of sak gene mutant ST398 infected mice.

\section{SAK promotes the activation of NLRP3 inflammasome}

We incubated BMDM of mice with bacterial culture supernatant or SAK protein and extracted the mRNA of the cells. Gene expression was detected by qRT-PCR after reverse transcription, and the results showed that SAK can effectively promote the expression of innate immune inflammation-related factors, such as cytokines (IL-17, TNF- $\alpha$, IL-1 $\beta$, IL-8) and NLRP3 inflammasome-related genes (Figure 4A-F). It should be noted that although the expression level of caspase- 1 and IL-1 $\beta$ genes increased 2fold after SAK protein treatment, we did not observe a significant increase in cleaved caspase-1 by western blot (Supplementary Figure 3). Therefore, whether the SAK protein can directly activate the NLRP3 inflammasome remains to be further studied. Moreover, what we can demonstrate is that in the presence of effective NLRP3 
activators (such as LPS or S. aureus culture filtrates), SAK can further promote the maturation of caspase- 1 and IL-1 $\beta$, and the difference between the wild-type strain and the sak mutant strain can also prove this point (Figure 4G, H). However, when the BMDM of caspase- $1^{-/-}$mice were incubated with bacterial supernatant or SAK protein, SAK could no longer promote the expression and maturation of IL-1 $\beta$ (Figure 4I, J). This indicates that the activation of the NLRP3 inflammasome plays an important role in the process of SAK promoting the maturation and release of IL-1 $\beta$.

\section{Inhibition of the NLRP3 inflammasome can reduce lung tissue damage caused by} ST398

We constructed a lethal mouse model of pneumonia to visually show the effect of SAK on the pathogenicity of ST398. The results showed that sak gene knockout can improve the survival rate of ST398-infected mice (Figure 5A). Correspondingly, HE staining of lung tissue sections of mice infected with wild-type ST398 showed a high degree of inflammatory cell infiltration, and immunohistochemistry analysis showed high levels of IL-1 $\beta$ (Figure 5B). In addition, the NLRP3 inflammasome can be inhibited by intraperitoneal injection of MCC950 (NLRP3 inhibitor) to mice. When mice were given NLRP3 inhibitors, their mortality decreased, and there was no significant difference in the survival rate of mice infected with wild-type ST398 and sak mutant strains (Figure 5C). This indicates that NLRP3 inflammasome activation plays an important role in SAK's promotion of the pathogenicity of ST398. Furthermore, the inhibitory effect of MCC950 on the NLRP3 inflammasome was confirmed by immunohistochemical staining of IL-1 $\beta$, and HE staining of lung tissue sections showed 
that inhibiting NLRP3 inflammasomes can reduce the recruitment of inflammatory cells and weaken the inflammatory damage caused by infection (Figure 5D).

SAK exerts a pro-inflammatory effect on THP1 cells, and can promote the production of ROS

Furthermore, we measured the cytokines released by human THP1 cells (human monocyte derived cell line) after stimulation with bacterial culture supernatant. Similar to the results in mouse models, SAK can also stimulate the release of inflammatory activation of the host inflammatory response by SAK is also applicable to human $S$. aureus infections. flux, production of ROS, and lysosomal damage ${ }^{12}$. In order to explore the mechanism by which SAK enhances the inflammatory response and promotes the activation of NLRP3 inflammasomes, we used potassium channel blocker glibenclamide, NADPH oxidase inhibitor apocynin, lysosome membrane stabilizer dexamethasone, and ROS scavenger N-acetyl-L-cysteine (NAC) to treat THP1 cells. The results showed that the addition of apocynin, dexamethasone and NAC could significantly reduce the expression of IL-1 $\beta$ gene in the SAK treatment group, and eliminate the expression difference between ST398 wild type and the sak mutant group (Figure 6D). In addition, only apocynin and NAC could inhibit the promoting effect of SAK on IL-1 $\beta$ secretion (Figure 6E). Apparently, the inhibition of $\mathrm{K}^{+}$efflux by glibenclamide does not change the effect of $S$. aureus culture supernatant on IL-1 $\beta$ secretion. Moreover, although the 
secretion of IL-1 $\beta$ can be significantly reduced by adding dexamethasone, there are still statistical differences between the wild type strain and the sak mutant strain (Figure 6E). Therefore, we consider that destroying lysosomes is not the main mechanism by which SAK promotes the pathogenicity of $S$. aureus. Since NADPH oxidase is proven to be an important source of ROS, we speculate that ROS production may play an important role in SAK's promotion of inflammation. Furthermore, by detecting the content of intracellular ROS, we found that SAK can significantly promote the generation of ROS, which can also be inhibited by NAC (Figure 6F).

\section{Discussion}

In our present study, we demonstrate that the expression level of SAK in CA-SA is significantly higher than that of HA-SA, and it plays an important role in acute necrotizing pneumonia by promoting the generation of ROS and activating NLRP3 inflammasomes. This may be a new mechanism for SAK to promote infection in addition to promoting fibrinolysis and neutralizing antimicrobial peptides. We have summarized the main functions of SAK and presented them in Figure 7.

Previous reports have shown that SAK can promote the activation of plasminogen in specific hosts, thus inhibiting the formation of biofilms and facilitating the spread of bacteria $^{24}$. The effect of SAK on biofilm formation is human plasminogendependent ${ }^{24,25}$, which is consistent with our results. It is worth noting that although the SAK highly expressed CA-SA biofilm formation ability is weak, there is no evidence that SAK can directly inhibit the formation of $S$. aureus biofilm. It has been found that the increased expression of virulence factors controlled by Agr is responsible for the 
high pathogenicity of ST398, and we speculate that the weak biofilm formation ability of CA-SA is more likely due to the high expression of the Agr system. In addition, the expression of SAK is also positively regulated by the Agr system. Furthermore, it has been proven that SAK can directly bind to murine CRAMP and promote fibrinolysis, while also playing a role in evading the host's innate immunity ${ }^{27}$. In this study, our data indicate that increased expression of sak may promote lung infection of ST398 isolates 249 in both wild-type and $C R A M P^{-/-}$mice. Since the effect of SAK on human plasminogen is species-specific, we speculate that promoting fibrinolysis and evading host innate immunity may not be the main mechanism of SAK aggravating pneumonia in ST398infected mice. consequences. In acute lung infections caused by high virulence CA-SA, uncontrolled excessive and long-term inflammation induced by virulence factors can lead to cell 256 death and tissue damage $e^{9,31,32}$, thereby hindering bacterial clearance ${ }^{11,33}$. Inflammation 257 is a double-edged sword for the body to resist microbial infections. Studies have shown of NLRP3 inflammasomes in mouse BMDM. Although we are still not sure whether 264 SAK can directly induce the maturation of caspase-1, in the presence of an effective 
NLRP3 inflammasome activator, SAK can further promote the maturation of caspase-

1 and the release of IL-1 $\beta$. Furthermore, the application of NLRP3 inflammasome inhibitors can significantly reduce the mortality of mice, and SAK no longer exhibits the effect of promoting infection. This may preliminarily explain the role of NLRP3 inflammasome activation in SAK's promotion of pathogenesis. In addition, SAK can also promote the release of cytokines from human THP1 cells, which indicates that the 271 activation of host inflammatory response by SAK is also suitable for human infections. seems that they may activate NLRP3 by inducing various cell signal transduction events, 275 including $\mathrm{K}^{+}$efflux, ROS production and lysosomal damage ${ }^{12}$. In this study, we found that SAK can promote the release of ROS in THP1 cells, and scavenging ROS or 277 inhibiting NADPH oxidase can eliminate the influence of SAK on IL-1 $\beta$ release. It is worth noting that in the RNA sequencing study of mouse lung tissue, we found that

279 SAK can promote the expression of the NLRP3 inflammasome upstream molecules, such as NF-kB and toll-like receptors, which may be another possible means of NLRP3 activation.

In previous studies, researchers tended to pay more attention to the promoting 283 effect of SAK on fibrinolysis. Our research found that SAK is also an effective proinflammatory factor for acute infections caused by CA-SA. This enriches our understanding of the function of SAK, and at the same time, we emphasize that in the pneumonia caused by CA-SA, the control of inflammation is as important as the anti- 
infection strategy. However, how SAK promotes the production of ROS and the mechanism of its pro-inflammatory effect needs to be confirmed by further studies.

In conclusion, this study preliminarily explored the pro-inflammatory role and

mechanism of SAK in acute $S$. aureus pneumonia. Our results emphasize the importance of controlling inflammation in acute lung infections, and also provides new ideas for the pathogenic mechanism of high virulence CA-SA.

\section{Methods}

\section{Bacterial isolates}

S. aureus strains were isolated from adult patients of Renji Hospital affiliated with Shanghai Jiaotong University. The concentration of antibiotics used when constructing gene knockout strains is as follows: ampicillin, $100 \mu \mathrm{g} / \mathrm{ml}$; chloramphenicol, $10 \mu \mathrm{g} / \mathrm{ml}$. Please see the Supplementary data-1 for more details.

\section{Measurement of SAK secretion by $S$. aureus}

Overnight cultures (12-15 hours) were diluted 1:100 into fresh TSB and incubated at $37^{\circ} \mathrm{C}$ with shaking at $200 \mathrm{rpm}$. Bacterial suspension at different time points was centrifuged (4000 rpm for 10 minutes at $4^{\circ} \mathrm{C}$ ) and the supernatant was collected after filtration. Human plasminogen (Roche) was mixed with supernatant and incubated at $37^{\circ} \mathrm{C}$ for 30 minutes, then a plasmin-specific chromogenic substrate $\mathrm{S}-2251(3 \mathrm{mmol} / \mathrm{L}$, amount of SAK secreted by the strains was read with a micro enzyme-linked immunosorbent assay (ELISA) autoreader (BioRad) at $405 \mathrm{~nm}$.

\section{Semiquantitative biofilm assay}


medium with $0.5 \%$ glucose or $10 \%$ plasma. Heparin anticoagulated human plasma was

311 provided voluntarily by healthy subjects. Mouse plasma came from the experimental

$312 \mathrm{C} 57 \mathrm{BL} / 6$ mice. Both human and mouse plasma were incubated at $56^{\circ} \mathrm{C}$ for 30 minutes

313 before use to inactivate complement. Wells were washed by sterile phosphate buffered

314 saline (PBS) after fixative for 1 hours, and then the biofilm was stained by crystal violet.

315 The floating stain was washed off with running water and the ability of biofilm

316 formation was reflected by the absorbance at $570 \mathrm{~nm}$ (Micro ELISA autoreader,

317 BioRad).

\section{Lung infection model in mice}

319 C57BL/6 wild-type or $C R A M P^{-/-}$(Jackson Laboratory) female mice (7-8 weeks old)

320 were used for the lung infection model. S. aureus strains were grown to mid-logarithmic

321 phase, washed once and then resuspended with sterile PBS at $5 \times 10^{6} \mathrm{CFU} / \mu \mathrm{l}$. Mice were 322 anesthetized with 2,2,2-tribromoethanol (3.75-5mg/25g), and then $40 \mu$ inoculum was 323 pipetted into the nares of the anesthetized mice slowly. For the fatal pneumonia model, $32450 \mathrm{mg} / \mathrm{kg}$ MCC950 sodium (NLRP3 inflammasome inhibitor, Selleck) or sterile PBS 325 was injected intraperitoneally into mice two hours before infection by $10^{9} \mathrm{CFU}\left(2 \times 10^{7}\right.$ $326 \mathrm{CFU} / \mu \mathrm{l}, 50 \mu \mathrm{l}) \mathrm{S}$. aureus. Please see the Supplementary data-1 for more details.

\section{Cell culture}

328 Mouse bone marrow derived macrophages (BMDM) were isolated from bone marrow 329 and differentiated for 7 days in RPMI 1640 medium supplemented with $10 \%$ fetal 330 bovine serum (FBS, Gibco), penicillin $(100 \mathrm{U} / \mathrm{ml})$ and streptomycin $(0.1 \mathrm{mg} / \mathrm{ml})$ and 20 ng/ml human M-CSF (R\&D Systems). THP1 cells (human monocytic leukemia cell 
line) were cultured in RPMI 1640 medium with FBS and penicillin and streptomycin.

Phorbol 12-myristate 13-acetate (100ng/ml, 24 hours) was used in differentiation of THP1 into macrophages. The additives or inhibitors used in in vitro experiments are as follows: purified SAK $(0.5 \mu \mathrm{g} / \mathrm{ml})$, lipopolysaccharide (LPS, $0.2 \mu \mathrm{g} / \mathrm{ml}) /$ ATP $(5 \mathrm{mM})$, potassium channel blocker glibenclamide $(10 \mu \mathrm{M}$, Sangon Biotech), NADPH oxidase inhibitor apocynin $(100 \mu \mathrm{M}$, Selleck), lysosome membrane stabilizer dexamethasone $(100 \mu \mathrm{M}$, Sangon Biotech), and reactive oxygen species (ROS) scavenger N-acetyl-Lcysteine (NAC, $10 \mu \mathrm{M}$, Sigma).

\section{Quantitative reverse-transcription (qRT) PCR}

Total RNA of $S$. aureus or cells was extracted, and complementary DNA (cDNA) was synthesized from total RNA by using the QuantiTect reverse transcription system (Qiagen). Amplification of the resulting cDNA sample utilized the QuantiTect SYBR green PCR kit (Qiagen). The 7500 Sequence Detector (Applied Biosystems) was used to perform reactions in MicroAmp Optical 96-well reaction plates. Oligonucleotides used in this study are presented in Supplementary data-1.

\section{RNA Sequencing}

Total RNA was extracted from lung tissues of mice (Qiagen). Nanodrop2000 was used to detect the concentration and purity of the extracted RNA, agarose gel electrophoresis was used to detect RNA integrity, and the Agilent 2100 was used to determine the RIN value. The library was constructed using the Illumina TruseqTM RNA sample prep Kit method, and finally sequenced on the Illumina Novaseq 6000 platform (Shanghai Majorbio Bio-pharm Technology Co., Ltd). 


\section{Western blot}

355

Overnight cultures (12-15 hours) were diluted 1:100 into fresh TSB and incubated at $37^{\circ} \mathrm{C}$ with shaking until stationary phase $\left(\mathrm{OD} 600_{\mathrm{nm}}=5.0\right) .100 \mu \mathrm{l}$ of culture filtrate was added into mouse BMDM cultured in a 6-well plate and incubate for 3 hours. The purified SAK $(0.5 \mu \mathrm{g} / \mathrm{ml})$ protein and lipopolysaccharide (LPS, $0.2 \mu \mathrm{g} / \mathrm{ml}) / \mathrm{ATP}(5 \mathrm{mM})$ were incubated with BMDM for 8 hours. Cells were collected after being washed by sterile PBS, and boiled in loading buffer. Then the samples were subjected to western blot experiment with caspase-1 (Abcam) and interleukin-1 $\beta$ (IL-1 $\beta$, Sangon Biotech) antibodies.

\section{Cytokine detection}

The level of mouse plasma cytokine was detected by Bio-Plex Pro Mouse Cytokine 23plex Assay in accordance with the manufacturer's instructions (Bio-Rad, Luminex $200^{\mathrm{TM}}$ System). IL-1 $\beta$ in the supernatant of mouse BMDM was measured by using ELISA kit (R\&D systems). IL-1 $\beta$ and tumor necrosis factor $\alpha$ (TNF- $\alpha$ ) released by THP1 cells were detected by Human IL-1 $\beta$ or TNF- $\alpha$ ELISA Kit (Sangon Biotech), and interleukin-17A (IL-17A) released by THP1 was measured by Human IL-17 Quantikine HS ELISA Kit (R\&D systems).

\section{Reactive Oxygen Species (ROS) Assay}

SAK $(0.5 \mu \mathrm{g} / \mathrm{ml})$ was added into the medium of THP1 cells, and NAC $(10 \mu \mathrm{M}$, Sigma) or PBS was added at the same time in 12 -well plates of cultured cells at $37^{\circ} \mathrm{C}$, and incubated for 5 hours, after which the fluorescent dye 2,7-Dichlorodi-hydrofluorescein diacetate (DCFH-DA, Sigma) was added to the cell culture medium and incubated for 
$30 \mathrm{~min}$ at $37^{\circ} \mathrm{C}$ in the dark. After washing off the excess dye, the fluorescence signal

377 was collected at an excitation wavelength of $480 \mathrm{~nm}$ and emission wavelength of 525

$378 \mathrm{~nm}$, and the cells placed under a fluorescence microscope for observation.

\section{Statistical Analysis}

380 Statistical analyses were performed with Graph-Pad Prism, version 8.0. The 381 comparison of survival curves was carried out by log-rank (Mantel-Cox) test. Unpaired

382 t-tests and two-way ANOVA were used to compare the differences between the 383 variables. All error bars in the graphs show standard deviation $( \pm \mathrm{SD}) . P<0.05$ was 384 regarded as statistically significant.

\section{Ethics Statement}

386 All animal experiments were performed in accordance with the laboratory animal care 387 and use guidelines of the Chinese Association for Laboratory Animal Sciences 388 (CALAS). Heparinized venous blood was donated voluntarily by healthy subjects and 389 the use of patient-derived isolates was approved by the ethics committee of Renji 390 Hospital, School of Medicine, Shanghai Jiao Tong University, Shanghai (RA-2020$391229)$.

\section{Data availability}

RNA-sequence data of this study have been submitted to NCBI (Accession number: SRR16107127, SRR16107128, SRR16107129, SRR16107130). The source data underlying the figures presented in this manuscript are provided in Supplementary

Data-2. Extra data are available from the corresponding author upon request. 


\section{References}

4001 McGuire, E., Boyd, A. \& Woods, K. Staphylococcus aureus Bacteremia. Clin Infect Dis 71,

401

402

403

404

405

406

407

408

409

410

411

412

413

414

415

416 2765-2766, doi:10.1093/cid/ciaa109 (2020).

2 Galar, A., Weil, A. A., Dudzinski, D. M., Munoz, P. \& Siedner, M. J. Methicillin-Resistant Staphylococcus aureus Prosthetic Valve Endocarditis: Pathophysiology, Epidemiology, Clinical Presentation, Diagnosis, and Management. Clinical microbiology reviews 32 , doi:10.1128/CMR.00041-18 (2019).

3 Bacterial and viral co-infections complicating severe influenza: Incidence and impact among 507 U.S. patients, 2013-14. Journal of Clinical Virology the Official Publication of the Pan American Society for Clinical Virology 80, 12-19 (2016).

4 Allou, N., Larsen, K., Dubernet, A., Traversier, N. \& Coolen-Allou, N. Co-infection in patients with hypoxemic pneumonia due to COVID-19 in Reunion Island. Medicine (Baltimore) 100, e24524 (2021).

5 Grousd, J. A., Rich, H. E. \& Alcorn, J. F. Host-Pathogen Interactions in Gram-Positive Bacterial Pneumonia. Clinical microbiology reviews 32, doi:10.1128/cmr.00107-18 (2019).

6 Otto, M. Basis of virulence in community-associated methicillin-resistant Staphylococcus aureus. Annu Rev Microbiol 64, 143-162, doi:10.1146/annurev.micro.112408.134309 (2010).

7 DuMont, A. L. et al. Staphylococcus aureus LukAB cytotoxin kills human neutrophils by targeting the CD11b subunit of the integrin Mac-1. Proc Natl Acad Sci U S A 110, 1079410799, doi:10.1073/pnas.1305121110 (2013).

8 Inoshima, I. et al. A Staphylococcus aureus pore-forming toxin subverts the activity of ADAM10 to cause lethal infection in mice. Nat Med 17, 1310-1314, doi:10.1038/nm.2451 (2011).

9 Kitur, K. et al. Toxin-induced necroptosis is a major mechanism of Staphylococcus aureus lung damage. PLoS pathogens 11, e1004820, doi:10.1371/journal.ppat.1004820 (2015).

10 Gomez, M. I. et al. Staphylococcus aureus protein A induces airway epithelial inflammatory responses by activating TNFR1. Nat Med 10, 842-848, doi:10.1038/nm1079 (2004).

11 Cohen, T. S. \& Prince, A. S. Bacterial pathogens activate a common inflammatory pathway through IFNlambda regulation of PDCD4. PLoS pathogens 9, e1003682, doi:10.1371/journal.ppat.1003682 (2013). Mechanisms of Activation and Regulation. International journal of molecular sciences $\mathbf{2 0}$, doi:10.3390/ijms20133328 (2019).

13 Lamkanfi, M. \& Dixit, V. M. Mechanisms and functions of inflammasomes. Ce//157, 10131022, doi:10.1016/j.cell.2014.04.007 (2014).

14 Chen, J. et al. RIP3 dependent NLRP3 inflammasome activation is implicated in acute lung injury in mice. J Trans/ Med 16, 233, doi:10.1186/s12967-018-1606-4 (2018).

15 Saleh, M. The machinery of Nod-like receptors: refining the paths to immunity and cell death. Immunological reviews 243, 235-246, doi:10.1111/j.1600-065X.2011.01045.x (2011). 

398 allows insight into community-associated methicillin-resistant Staphylococcus aureus evolution. Genome Med 10, 5, doi:10.1186/s13073-018-0514-9 (2018). Dai, Y. et al. Decreasing methicillin-resistant Staphylococcus aureus (MRSA) infections is attributable to the disappearance of predominant MRSA ST239 clones, Shanghai, 2008 2017. Emerg Microbes Infect 8, 471-478, doi:10.1080/22221751.2019.1595161 (2019).

Chuang, Y.-Y. \& Huang, Y.-C. Molecular epidemiology of community-associated meticillin-resistant Staphylococcus aureus in Asia. The Lancet Infectious Diseases 13, 698 708, doi:10.1016/s1473-3099(13)70136-1 (2013).

9 Wang, Y. et al. Role of the ESAT-6 secretion system in virulence of the emerging community-associated Staphylococcus aureus lineage ST398. Sci Rep 6, 25163, doi:10.1038/srep25163 (2016).

Dai, Y. et al. A Novel ESAT-6 Secretion System-Secreted Protein EsxX of CommunityAssociated Staphylococcus aureus Lineage ST398 Contributes to Immune Evasion and Virulence. Front Microbio/ 8, 819, doi:10.3389/fmicb.2017.00819 (2017). Infect Genet Evo/ 21, 593-601, doi:10.1016/j.meegid.2013.04.022 (2014).

Coombs, G. W., Baines, S. L., Howden, B. P., Swenson, K. M. \& O'Brien, F. G. Diversity of bacteriophages encoding Panton-Valentine leukocidin in temporally and geographically related Staphylococcus aureus. PLOS One 15, e0228676, doi:10.1371/journal.pone.0228676 (2020).

van Wamel, W. J., Rooijakkers, S. H., Ruyken, M., van Kessel, K. P. \& van Strijp, J. A. The innate immune modulators staphylococcal complement inhibitor and chemotaxis inhibitory protein of Staphylococcus aureus are located on beta-hemolysin-converting bacteriophages. J Bacterio/188, 1310-1315, doi:10.1128/JB.188.4.1310-1315.2006 (2006). Kwiecinski, J. et al. Staphylokinase Control of Staphylococcus aureus Biofilm Formation and Detachment Through Host Plasminogen Activation. J Infect Dis 213, 139-148, doi:10.1093/infdis/jiv360 (2016).

Bokarewa, M. I., Jin, T. \& Tarkowski, A. Staphylococcus aureus: Staphylokinase. Int J Biochem Cel/ Bio/ 38, 504-509, doi:10.1016/j.biocel.2005.07.005 (2006).

26 Jin, T. et al. Staphylococcus aureus resists human defensins by production of staphylokinase, a novel bacterial evasion mechanism. J Immunol 172, 1169-1176, doi:10.4049/jimmunol.172.2.1169 (2004).

Braff, M. H., Jones, A. L., Skerrett, S. J. \& Rubens, C. E. Staphylococcus aureus exploits cathelicidin antimicrobial peptides produced during early pneumonia to promote staphylokinase-dependent fibrinolysis. J Infect Dis 195, 1365-1372, doi:10.1086/513277 (2007).

Verkaik, N. J. et al. Immune evasion cluster-positive bacteriophages are highly prevalent among human Staphylococcus aureus strains, but they are not essential in the first stages of nasal colonization. Clin Microbiol Infect 17, 343-348, doi:10.1111/j.14690691.2010.03227.x (2011).

Van, A. H., Van, D. P. \& Coenye, T. Molecular mechanisms of antimicrobial tolerance and resistance in bacterial and fungal biofilms. Trends in Microbiology 22, 326-333 (2014).

30 Boles, B. R., Horswill, A. R. \& Cossart, P. agr-Mediated Dispersal of Staphylococcus aureus 
Biofilms. PLoS pathogens 4, e1000052 (2008).

31 Pasparakis, M. \& Vandenabeele, P. Necroptosis and its role in inflammation. Nature 517, 311-320, doi:10.1038/nature14191 (2015).

32 Pinkerton, J. W. et al. Inflammasomes in the lung. Mol Immunol 86, 44-55, doi:10.1016/j.molimm.2017.01.014 (2017).

33 Kapetanovic, R. et al. Contribution of NOD2 to lung inflammation during Staphylococcus

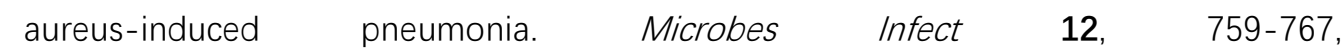
doi:10.1016/j.micinf.2010.05.003 (2010).

34 He, Y., Hara, H. \& Núñez, G. Mechanism and Regulation of NLRP3 Inflammasome Activation. Trends in biochemical sciences 41, 1012-1021, doi:10.1016/j.tibs.2016.09.002 (2016).

\section{Acknowledgements}

This work was supported by the National Natural Science Foundation of China (81861138043, 81800657), the Shanghai Committee of Science and Technology, China (19JC1413005), Clinical Research Plan of SHDC (SHDC2020CR3006A) and Shanghai Sailing Program (21YF1425500).

\section{Author contributions}

Y.W. and N. Z. performed the experiments and wrote the manuscript. Y. J. and Y.L. analyzed the RNA-sequence data. L.Z. and L.H. performed the statistical analysis. Q.L. and M.L. designed the experiments and revised the manuscript. All authors reviewed the manuscript.

\section{Competing interests}

The authors declare that they have no conflict of interest. 

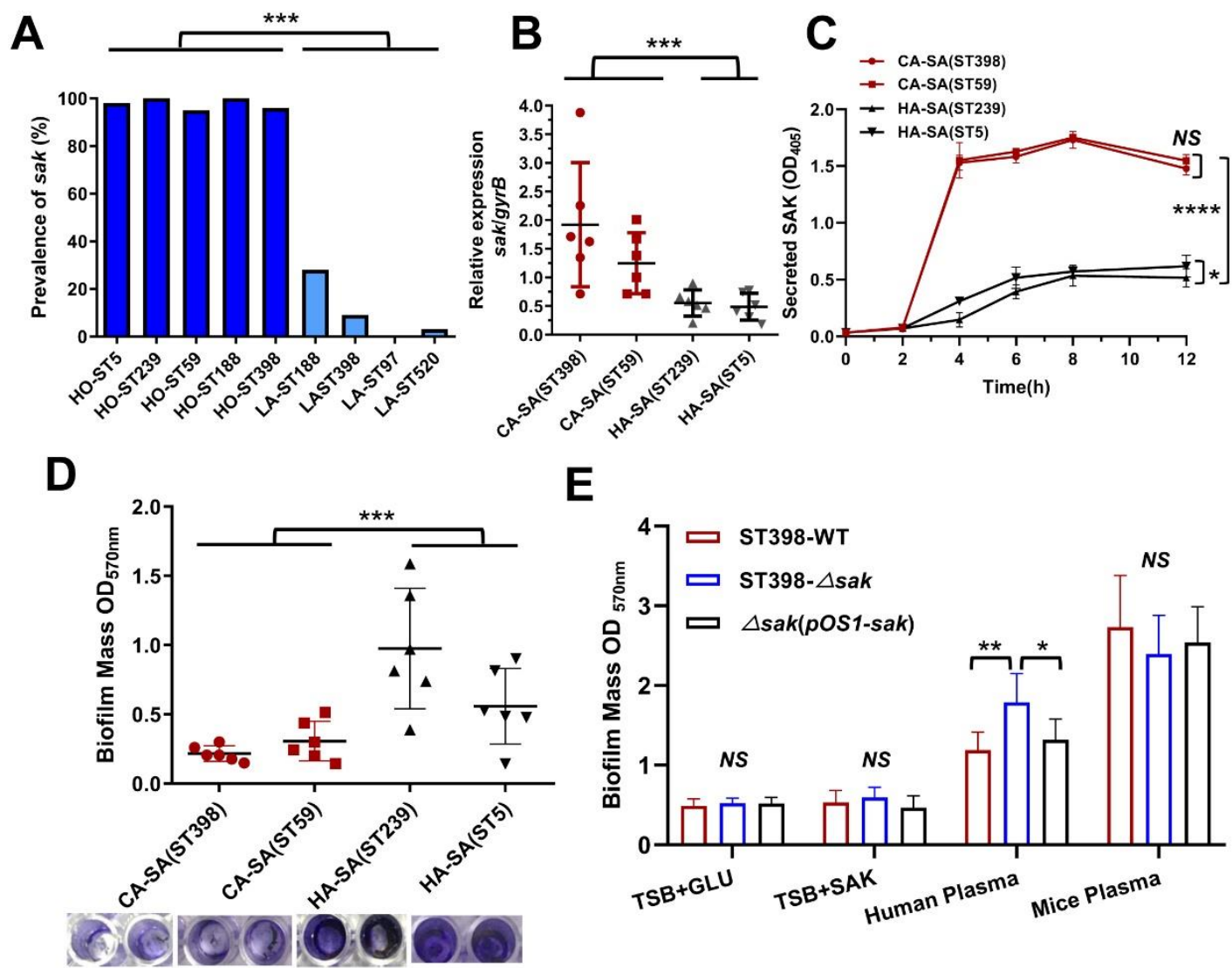

E

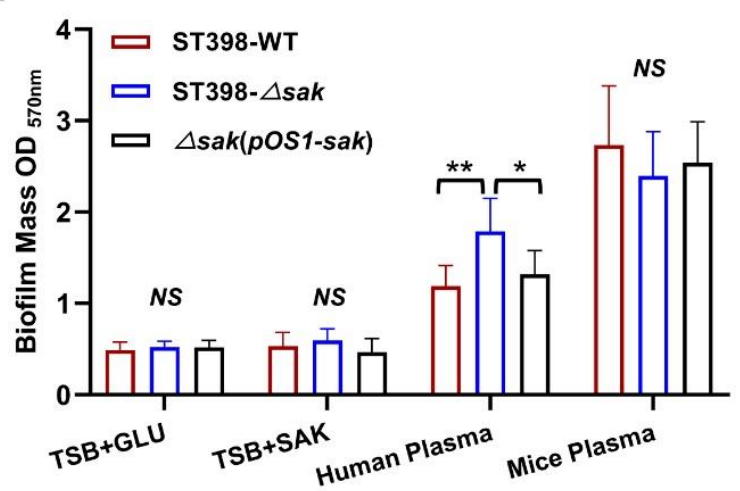

Figure 1. The expression level of SAK and its effect on the formation of biofilm. A, The prevalence of $s a k$ gene in $S$. aureus (SA) epidemic isolates. Deep blue: humansadapted (HO) isolates; Light blue: livestock-adapted (LA) isolates. Unpaired $t$ test was used for statistical analyses between HO-SA and LA-SA. B, qRT-PCR analysis of sak gene expression in randomly selected clinical CA-SA (ST398, ST59) and HA-SA (ST239, ST5) isolates, at $4 \mathrm{~h}$ of in vitro growth. Relative mRNA levels were calculated using gyrb as control and expressed as $2^{\wedge}(-\Delta \Delta \mathrm{Ct})$. Unpaired $t$ test was used for statistical analyses between CA-SA and HA-SA. C, Measurement of SAK secretion at different incubation times. Two-way ANOVA was used for statistical analyses. D, Biofilm formation ability of CA-SA (ST398, ST59) and HA-SA (ST239, ST5). Unpaired $t$ test was used for statistical analyses between CA-SA and HA-SA. E, The effect of adding glucose (GLU), SAK protein, human plasma and mice plasma on the biofilm formation of ST398-WT, sak deletion mutant isolate and the complemented isolate. Two-way ANOVA was used for statistical analyses. All data in figure 1 are presented as mean $\pm \mathrm{SD}$ and $* P<0.05, * * P<0.01$, *** $P<0.001$. 


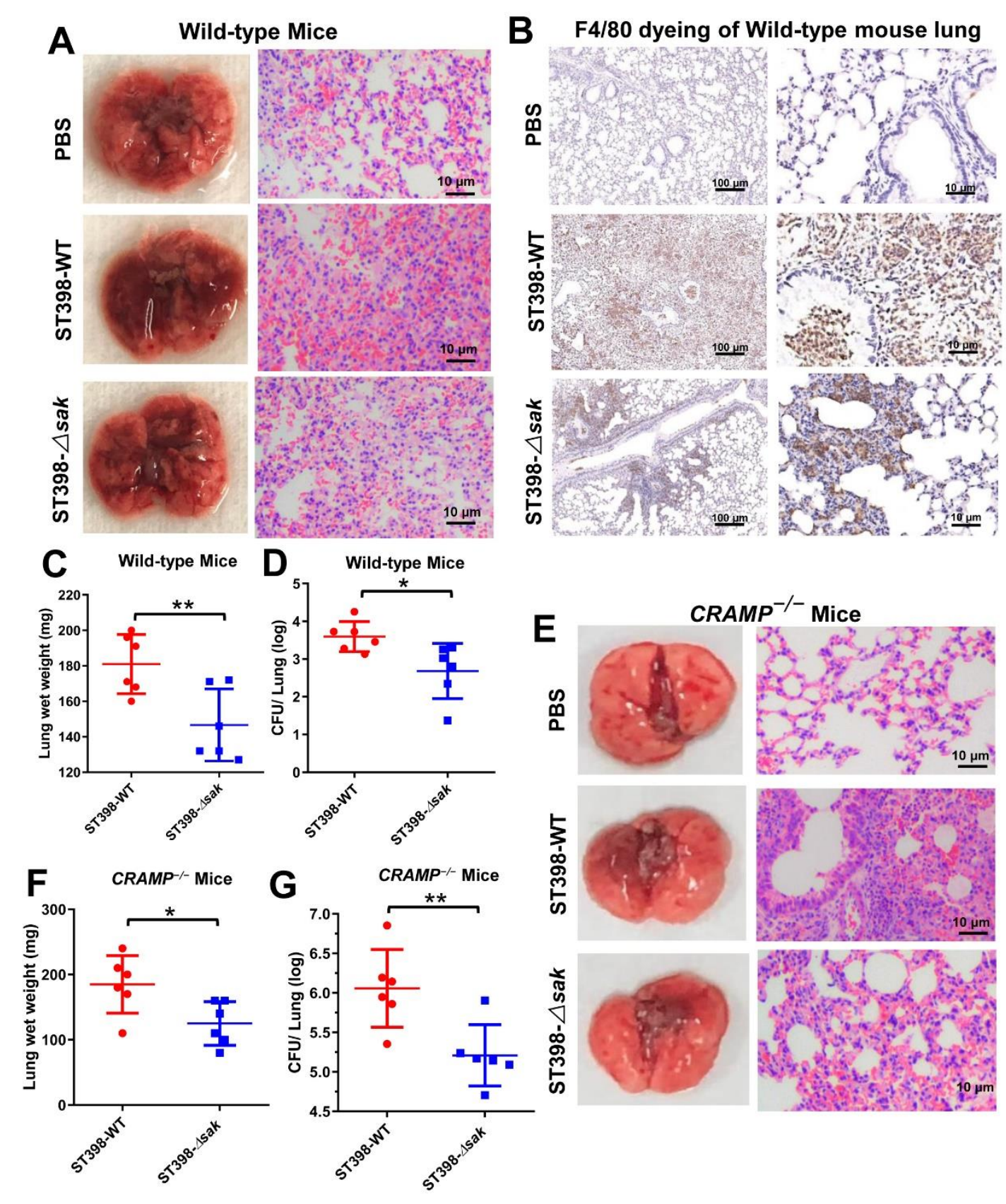

Figure 2. The mouse model of pneumonia shows the role of SAK in ST398 infection. A-G, $2 \times 10^{8}$ CFU of $S$. aureus was pipetted into the nares of anesthetized C57BL/ 6 wildtype mice (A-D) and $C R A M P^{-/-}$mice $(\mathrm{E}-\mathrm{G})(\mathrm{n}=7)$. A, E, Photographs of lungs and corresponding $\mathrm{H} \& \mathrm{E}$ stained sections of mice 48 hours after infection. B, Immunohistochemical staining (F4/80) was performed on the lung tissue of infected mice to observe the infiltration of macrophages. $\mathrm{C}, \mathrm{F}$, Lung wet weight of infected mice. $\mathrm{D}, \mathrm{G}$, The left lung was homogenized and plated on TSB agar for CFU determination. Unpaired $t$ test was used for statistical analyses between ST398-WT and sak deletion mutant isolate infected mice. Data are presented as mean $\pm \mathrm{SD}$ and $* P<0.05, * * P<0.01$. 

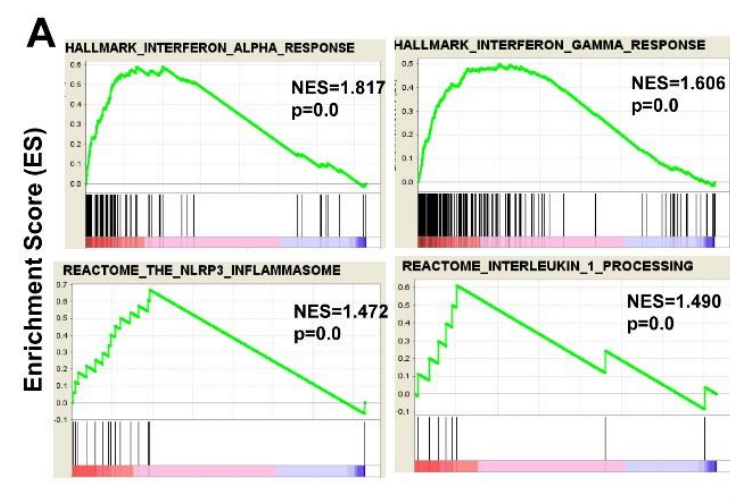

B
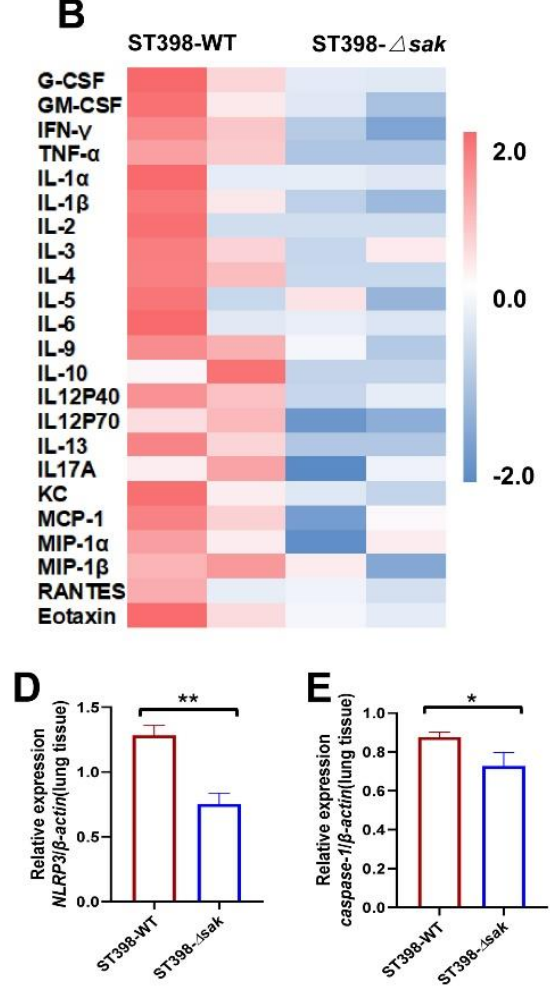

C
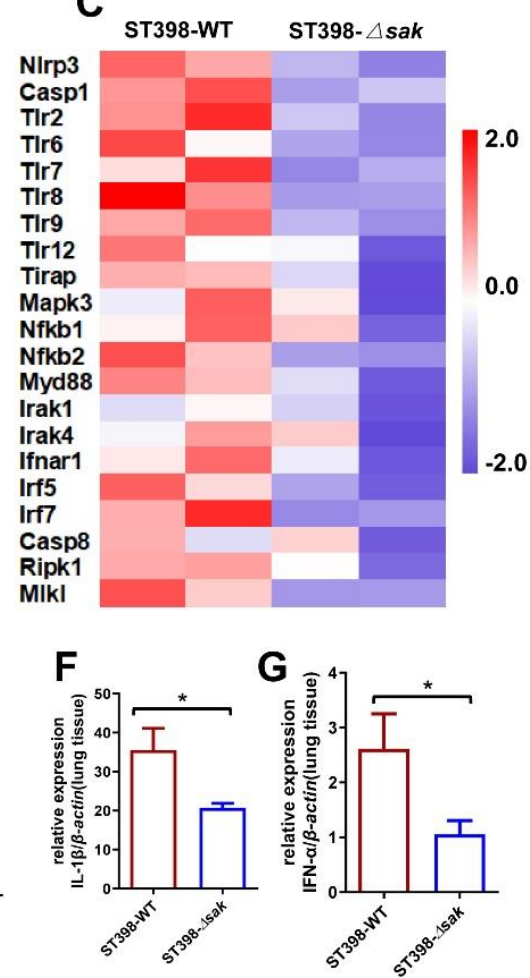

Figure 3. The activation of innate immune inflammatory response in lung infection of mice. A-G, Total RNA was extracted from lung tissues of mice infected by ST398WT and sak deletion mutants. A, GSEA analysis identified cytokine related genes (IFN$\alpha$, IFN- $\gamma$, IL-6, IL-1), complement related genes and NLRP3 inflammasome pathway enrichment in the wild type infected mice. B. The level of mouse plasma cytokine was detected by Bio-Plex Pro Mouse Cytokine 23-plex Assay. The data were normalized by the $\mathrm{Z}$ score according to the standard deviation from the mean. $\mathrm{C}$, Comparison of the expression levels of NLRP3 inflammasome activation-related molecules between the two groups. The data were normalized by the $\mathrm{Z}$ score according to the standard deviation from the mean. D-G, qRT-PCR analysis of NLRP3, caspase-1, IL-1 $\beta$, and IFN- $\alpha$ in the lung tissues of wild-type or sak deletion mutants infected mice. Relative mRNA levels were calculated using $\beta$-actin as control and expressed as $2^{\wedge}(-\Delta \Delta \mathrm{Ct})$. Unpaired $t$ test was used for statistical analyses and data are presented as mean $\pm \mathrm{SD}$ and $* P<0.05, * * P<0.01$. See Supplementary data- 1 for the abbreviated list of cytokines or genes in Figure $3 \mathrm{~B}$ and Figure $3 \mathrm{C}$. 


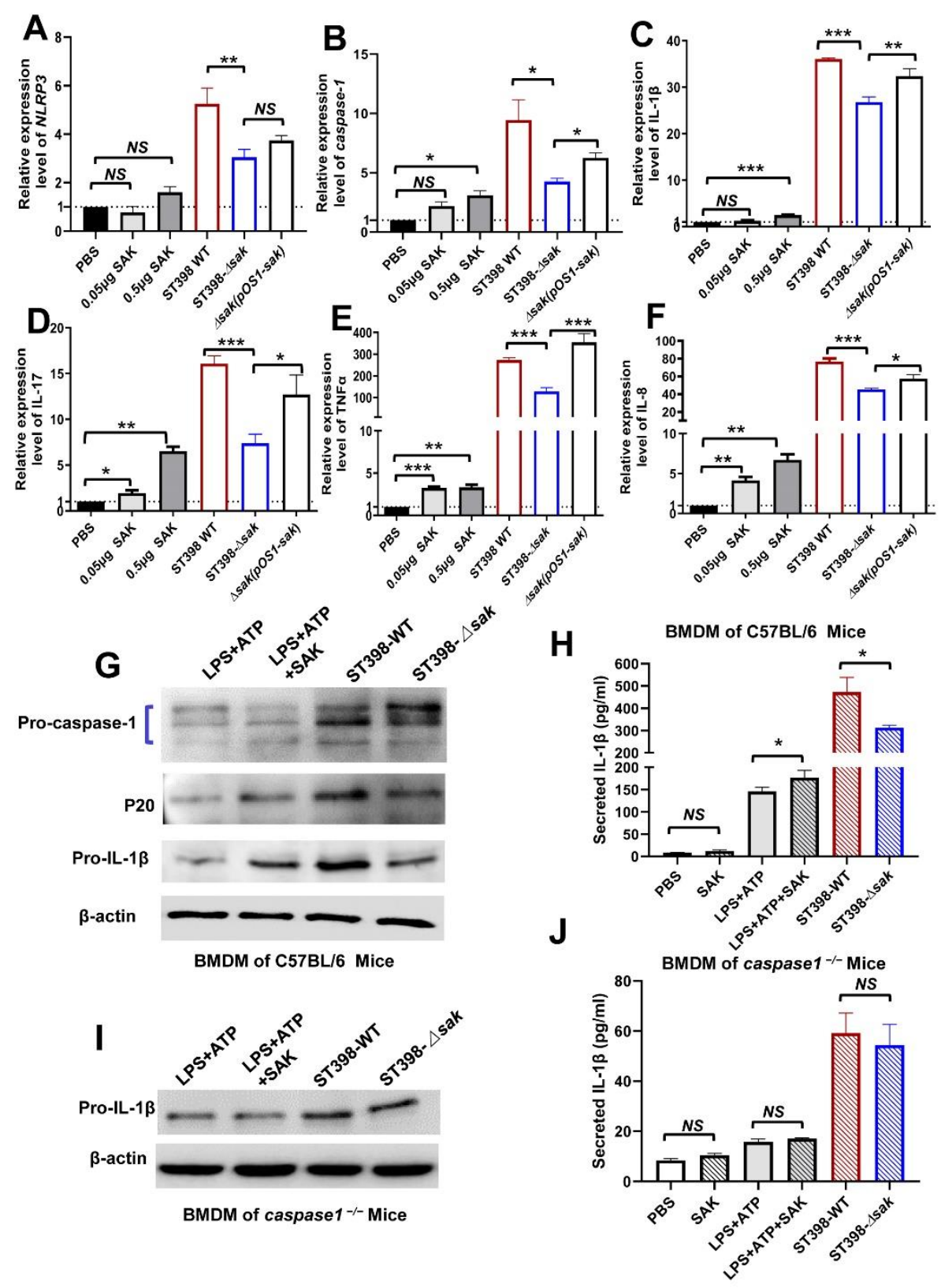

Figure 4. The promotion of SAK on NLRP3 inflammasome activation and cytokine expression. A-J, BMDM are incubated with bacterial secretion supernatant or SAK protein. A-F, The mRNA of the cells was extracted and gene expression relative to the negative control group was detected by qRT-PCR after reverse transcription. Relative mRNA levels were calculated using $\beta$-actin as internal control and expressed as $2^{\wedge}(-\Delta \Delta \mathrm{Ct})$. Unpaired t-test was used to compare the differences between the variables. G: Immunoblot analysis of the cell lysates from BMDM of wild-type mice. $\mathrm{H}$ : Secreted IL-1 $\beta$ from BMDM was detected by ELISA. Unpaired t-test was used to compare the differences between the variables. I: Immunoblot analysis of the cell lysates from BMDM of caspase $^{-/}$mice. J: Secreted IL-1 $\beta$ from BMDM of caspase $^{-/-}$ mice was detected by ELISA. Unpaired t-test was used to compare the differences between the variables. All data are presented as mean $\pm \mathrm{SD}$ and $* P<0.05, * * P<0.01$, *** $P<0.001$. 

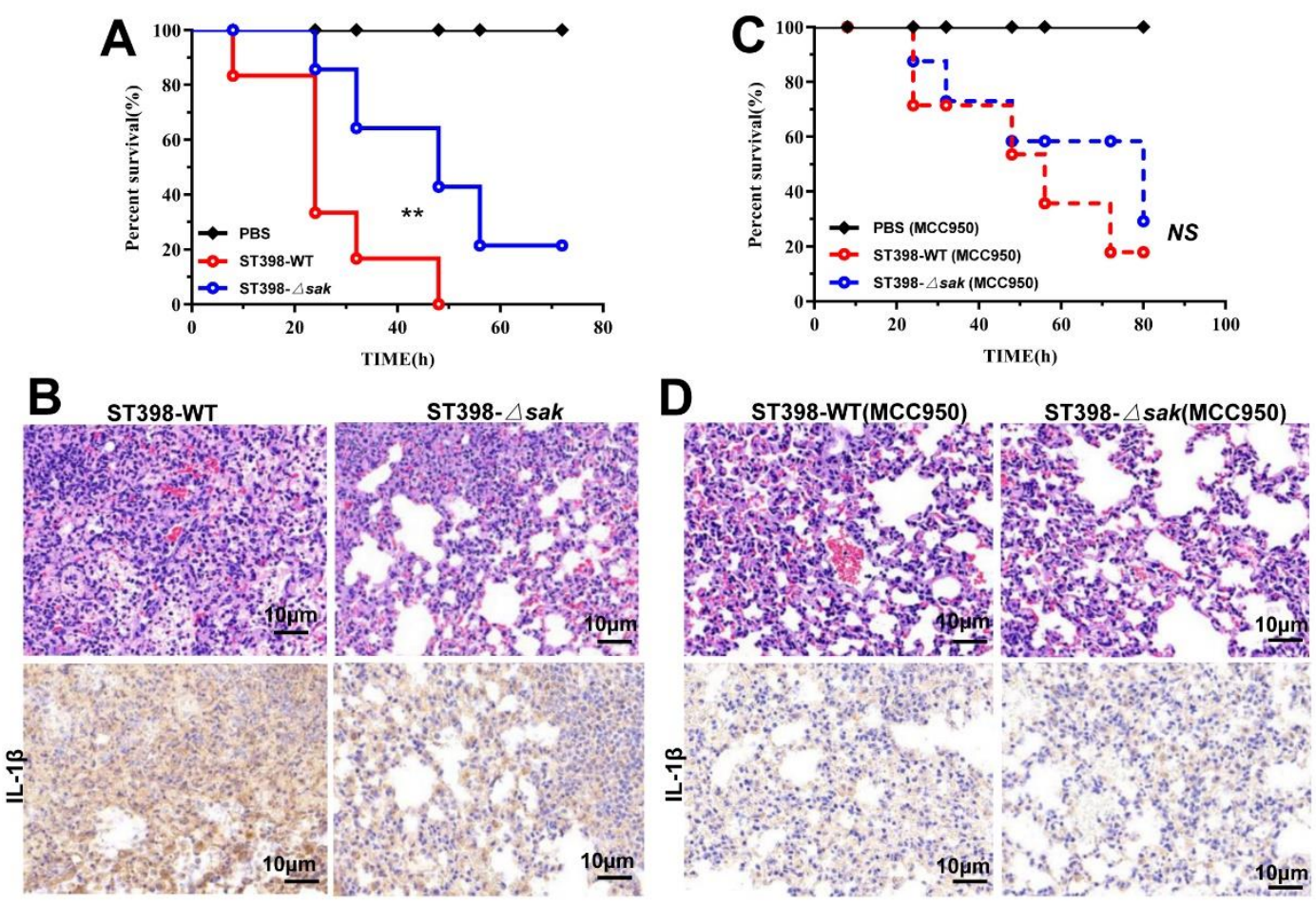

Figure 5. Inhibition of the NLRP3 inflammasome can reduce lung infection in mice. A-D: $10^{9} \mathrm{CFU}$ of $S$. aureus was pipetted into the nares of anesthetized C57BL/6 mice $(\mathrm{n}=6)$, and $50 \mathrm{mg} / \mathrm{kg}$ MCC950 sodium (NLRP3 inflammasome inhibitor) or PBS was injected intraperitoneally to mice two hours before infection. A, C, Survival curves were compared using a log-rank (Mantel-Cox) test. B, D, Tissue sections for HE staining and immunohistochemical staining (IL-1 $\beta$ ). ${ }^{* *} P<0.01$ 


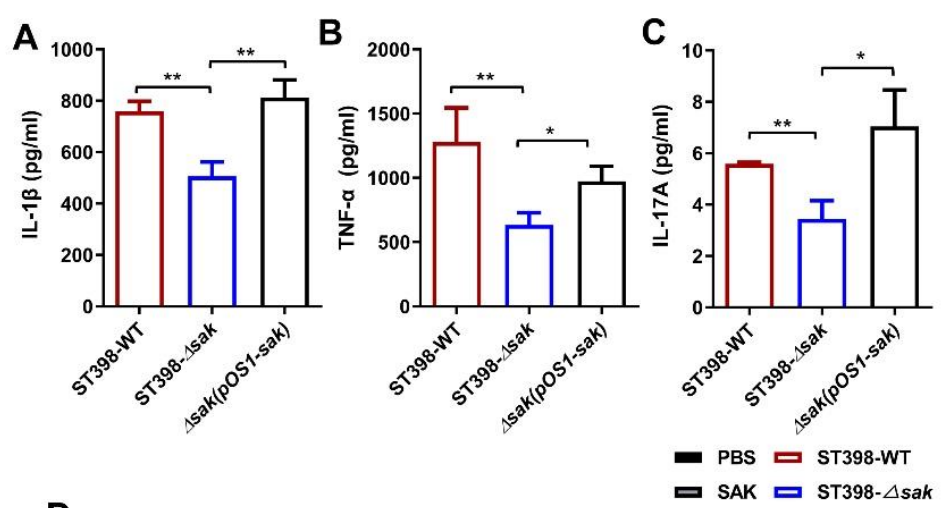

D

口 SAK 口 $\mathrm{ST} 398-\Delta s a k$
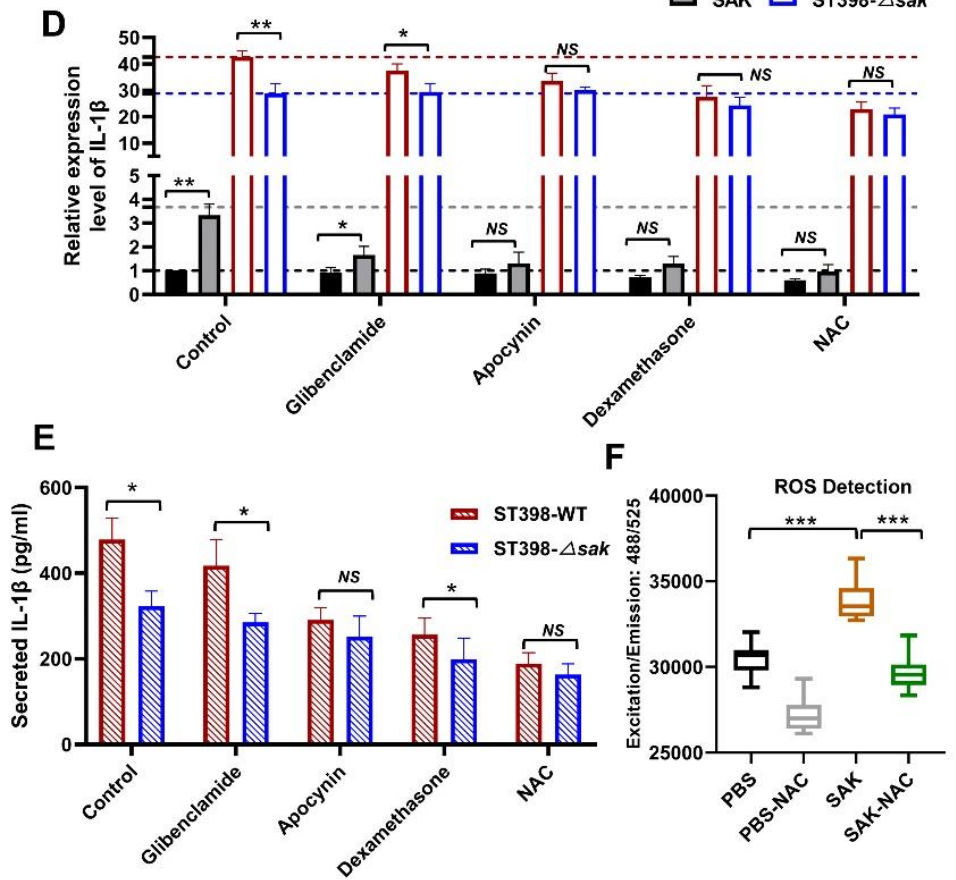

Figure 6. SAK exerts a pro-inflammatory effect on THP1 cells, and can promote the release of IL-1 $\beta$ by increasing the production of ROS. A-C, Cultured THP1 cells (human monocyte derived cell line) were incubated with bacterial secretion supernatant. The physiologically relevant concentrations of specific cytokine proteins in THP1 cell culture supernatants were detected by ELISA kit. Unpaired t-test was used to compare the differences between the variables. D-E, Detection of the role of ion flux, ROS production and lysosomal damage in the promotion of IL-1 $\beta$ expression and secretion by SAK. D. The expression level of IL$1 \beta$ gene in THP 1 cells treated with SAK protein or ST398 culture filtrate relative to the negative control group. Relative mRNA levels were calculated using GAPDH as internal control and expressed as $2^{\wedge}(-\Delta \Delta C t)$. Unpaired t-test was used to compare the differences between the variables. E. The secretion of IL-1 $\beta$ in THP1 cells treated with ST398 culture filtrate was detected by ELISA. Unpaired t-test was used to compare the differences between the variables. F. Reactive Oxygen Species Assay. The fluorescence signal was collected at the excitation wavelength of $488 \mathrm{~nm}$ and the emission wavelength of $525 \mathrm{~nm}$. Unpaired t-test was used to compare the differences between the variables. All data are presented as mean $\pm \mathrm{SD}$ and $* P<0.05, * * P<0.01, * * *$ $P<0.001$. 


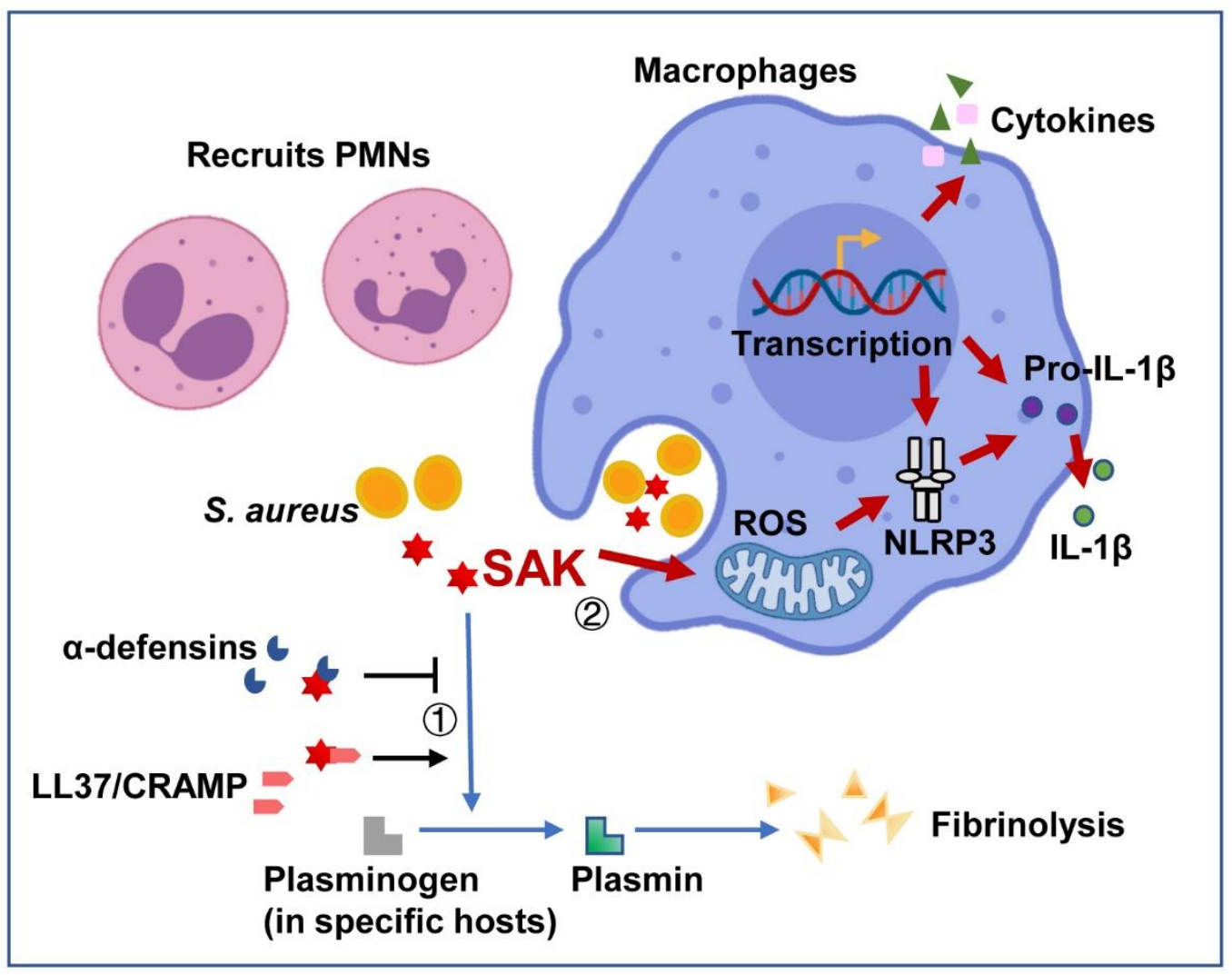

607

608

609

610

611

612

613

614

615

616

617

618

619

620

621

622

623

624

Figure 7. The role of SAK in Staphylococcus aureus infection.

(1) SAK can neutralize AMPs and regulate fibrinolysis.

(2) SAK promotes transcription of inflammation-related genes and activation of NLRP3 inflammasome.

Research has shown that SAK can promote the activation of plasminogen in specific hosts (human, rabbits, and sheep) and exert its fibrinolytic function (blue arrows). The epithelium expresses antimicrobial peptides to kill extracellular bacteria. Studies have shown that SAK can bind to $\alpha$-defensins, human cathelicidin LL-37 and mouse CRAMP to regulate fibrinolysis and evade innate immunity defenses (black arrows). In this study, we demonstrate that SAK plays an important role in acute necrotizing pneumonia by promoting the generation of ROS and activating NLRP3 inflammasomes (red arrows). Furthermore, we speculate through preliminary studies that SAK can promote the transcription of inflammatory response-related molecules and the release of cytokines, help recruit polymorphonuclear leukocytes (PMNs) and contribute to a more active state of inflammatory response in the host. This figure was drawn by using BioRender (https://app.biorender.com/). 


\section{Supplementary Files}

This is a list of supplementary files associated with this preprint. Click to download.

- Supplementarydata1.docx

- Supplementarydata2.xlsx 